\title{
SUPERADDITIVITY OF SOME FUNCTIONALS ASSOCIATED WITH JENSEN'S INEQUALITY FOR CONVEX FUNCTIONS ON LINEAR SPACES WITH APPLICATIONS
}

\author{
S. S. DRAGOMIR
}

(Received 17 September 2009)

\section{Abstract}

Some new results related to Jensen's celebrated inequality for convex functions defined on convex sets in linear spaces are given. Applications for norm inequalities in normed linear spaces and $f$-divergences in information theory are provided as well.

2000 Mathematics subject classification: primary 26D15; secondary 94A17.

Keywords and phrases: convex functions, Jensen's inequality, norms, mean $f$-deviation, $f$-divergences.

\section{Introduction}

The Jensen inequality for convex functions plays a crucial role in the theory of inequalities due to the fact that other inequalities such as the generalized triangle inequality, the arithmetic-geometric mean inequality, Hölder and Minkowski inequalities, Ky Fan's inequality and so on can be obtained as particular cases of it.

Let $C$ be a convex subset of the linear space $X$ and $f$ a convex function on $C$. If $I$ denotes a finite subset of the set $\mathbb{N}$ of natural numbers, $x_{i} \in C, p_{i} \geq 0$ for $i \in I$ and $P_{I}:=\sum_{i \in I} p_{i}>0$, then

$$
f\left(\frac{1}{P_{I}} \sum_{i \in I} p_{i} x_{i}\right) \leq \frac{1}{P_{I}} \sum_{i \in I} p_{i} f\left(x_{i}\right),
$$

is well known in the literature as Jensen's inequality.

We introduce the following notation (see also [14]):

$$
\begin{aligned}
F(C, \mathbb{R}) & :=\text { the linear space of all real functions on } C, \\
F^{+}(C, \mathbb{R}) & :=\{f \in F(C, \mathbb{R}): f(x)>0 \text { for all } x \in C\}, \\
P_{f}(\mathbb{N}) & :=\{I \subset \mathbb{N}: I \text { is finite }\}, \\
J(\mathbb{R}) & :=\left\{p=\left\{p_{i}\right\}_{i \in \mathbb{N}}, p_{i} \in \mathbb{R} \text { are such that } P_{I} \neq 0 \text { for all } I \in P_{f}(\mathbb{N})\right\},
\end{aligned}
$$

(C) 2010 Australian Mathematical Publishing Association Inc. 0004-9727/2010 \$16.00 


$$
\begin{aligned}
& J^{+}(\mathbb{R}):=\left\{p \in J(\mathbb{R}): p_{i} \geq 0 \text { for all } i \in \mathbb{N}\right\}, \\
& J_{*}(C):=\left\{x=\left\{x_{i}\right\}_{i \in \mathbb{N}}: x_{i} \in C \text { for all } i \in \mathbb{N}\right\}
\end{aligned}
$$

and

$$
\operatorname{Conv}(C, \mathbb{R}):=\text { the cone of all convex functions defined on } C .
$$

In [14] the authors considered the following functional associated with the Jensen inequality:

$$
J(f, I, p, x):=\sum_{i \in I} p_{i} f\left(x_{i}\right)-P_{I} f\left(\frac{1}{P_{I}} \sum_{i \in I} p_{i} x_{i}\right)
$$

where $f \in F(C, \mathbb{R}), I \in P_{f}(\mathbb{N}), p \in J^{+}(\mathbb{R}), x \in J_{*}(C)$. They established some quasi-linearity and monotonicity properties and applied the results obtained to norm and mean inequalities.

The following result concerning the properties of the functional $J(f, I, \cdot, x)$ as a function of weights holds (see [14, Theorem 2.4]).

Theorem 1.1. Let $f \in \operatorname{Conv}(C, \mathbb{R}), I \in P_{f}(\mathbb{N})$ and $x \in J_{*}(C)$.

(i) If $p, q \in J^{+}(\mathbb{R})$ then

$$
J(f, I, p+q, x) \geq J(f, I, p, x)+J(f, I, q, x)(\geq 0),
$$

that is, $J(f, I, \cdot, x)$ is superadditive on $J^{+}(\mathbb{R})$.

(ii) If $p, q \in J^{+}(\mathbb{R})$ with $p \geq q$, meaning that $p_{i} \geq q_{i}$ for each $i \in \mathbb{N}$, then

$$
J(f, I, p, x) \geq J(f, I, q, x)(\geq 0),
$$

that is, $J(f, I, \cdot, x)$ is monotonic nondecreasing on $J^{+}(\mathbb{R})$.

The behavior of this functional as an index set function is incorporated in the following result (see [14, Theorem 2.1]).

Theorem 1.2. Let $f \in \operatorname{Conv}(C, \mathbb{R}), p \in J^{+}(\mathbb{R})$ and $x \in J_{*}(C)$.

(i) If $I, H \in P_{f}(\mathbb{N})$ with $I \cap H=\varnothing$, then

$$
J(f, I \cup H, p, x) \geq J(f, I, p, x)+J(f, H, p, x)(\geq 0),
$$

that is, $J(f, \cdot, p, x)$ is superadditive as an index set function on $P_{f}(\mathbb{N})$.

(ii) If $I, H \in P_{f}(\mathbb{N})$ with $H \subset I$, then

$$
J(f, I, p, x) \geq J(f, H, p, x)(\geq 0),
$$

that is, $J(f, \cdot, p, x)$ is monotonic nondecreasing as an index set function on $P_{f}(\mathbb{N})$. 
As pointed out in [14], the above Theorem 1.2 is a generalization of the VasićMijalković result for convex functions of a real variable obtained in [24] and therefore creates the possibility of obtaining vectorial inequalities as well.

For applications of the above results to logarithmic convex functions, to norm inequalities, in relation to the arithmetic-geometric mean inequality and to other classical results, see [6-10, 12-15, 19, 22].

Motivated by the above results, we introduce in the present paper a more general functional, establish its main properties and use it for some particular cases that provide inequalities of interest. Applications to norm inequalities in normed linear spaces and $f$-divergences in information theory are provided as well.

\section{Some superadditivity properties for the weights}

We consider the more general functional

$$
D(f, I, p, x ; \Phi):=P_{I} \Phi\left[\frac{1}{P_{I}} \sum_{i \in I} p_{i} f\left(x_{i}\right)-f\left(\frac{1}{P_{I}} \sum_{i \in I} p_{i} x_{i}\right)\right],
$$

where $f \in \operatorname{Conv}(C, \mathbb{R}), I \in P_{f}(\mathbb{N}), p \in J^{+}(\mathbb{R}), x \in J_{*}(C)$ and $\Phi:[0, \infty) \rightarrow \mathbb{R}$ is a function whose properties will determine the behavior of the functional $D$ as follows. Obviously, for $\Phi(t)=t$ we recapture from $D$ the functional $J$ considered in [14].

First of all we observe that, by Jensen's inequality, the functional $D$ is well defined and positive homogeneous in the third variable, that is,

$$
D(f, I, \alpha p, x ; \Phi)=\alpha D(f, I, p, x ; \Phi),
$$

for any $\alpha>0$ and $p \in J^{+}(\mathbb{R})$.

The following result concerning the superadditivity and monotonicity of the functional $D$ as a function of weights holds.

Theorem 2.1. Let $f \in \operatorname{Conv}(C, \mathbb{R}), I \in P_{f}(\mathbb{N})$ and $x \in J_{*}(C)$. Assume that $\Phi$ : $[0, \infty) \rightarrow \mathbb{R}$ is monotonic nondecreasing and concave.

(i) If $p, q \in J^{+}(\mathbb{R})$ then

$$
D(f, I, p+q, x ; \Phi) \geq D(f, I, p, x ; \Phi)+D(f, I, q, x ; \Phi),
$$

that is, $D$ is superadditive as a function of weights.

(ii) If $p, q \in J^{+}(\mathbb{R})$ with $p \geq q$, meaning that $p_{i} \geq q_{i}$ for each $i \in \mathbb{N}$ and $\Phi$ : $[0, \infty) \rightarrow[0, \infty)$, then

$$
D(f, I, p, x ; \Phi) \geq D(f, I, q, x ; \Phi)(\geq 0),
$$

that is, D is monotonic nondecreasing as a function of weights. 
PROOF.

(i) Let $p, q \in J^{+}(\mathbb{R})$. By the convexity of the function $f$ on $C$,

$$
\begin{aligned}
\frac{1}{P_{I}+Q_{I}} \sum_{i \in I}\left(p_{i}+q_{i}\right) f\left(x_{i}\right)-f\left(\frac{1}{P_{I}+Q_{I}} \sum_{i \in I}\left(p_{i}+q_{i}\right) x_{i}\right) \\
=\frac{P_{I}\left(\frac{1}{P_{I}} \sum_{i \in I} p_{i} f\left(x_{i}\right)\right)+Q_{I}\left(\frac{1}{Q_{I}} \sum_{i \in I} q_{i} f\left(x_{i}\right)\right)}{P_{I}+Q_{I}} \\
\quad-f\left(\frac{P_{I}\left(\frac{1}{P_{I}} \sum_{i \in I} p_{i} x_{i}\right)+Q_{I}\left(\frac{1}{Q_{I}} \sum_{i \in I} q_{i} x_{i}\right)}{P_{I}+Q_{I}}\right) \\
\geq \quad \frac{P_{I}\left(\frac{1}{P_{I}} \sum_{i \in I} p_{i} f\left(x_{i}\right)\right)+Q_{I}\left(\frac{1}{Q_{I}} \sum_{i \in I} q_{i} f\left(x_{i}\right)\right)}{P_{I}+Q_{I}} \\
\quad-\frac{P_{I} f\left(\frac{1}{P_{I}} \sum_{i \in I} p_{i} x_{i}\right)+Q_{I} f\left(\frac{1}{Q_{I}} \sum_{i \in I} q_{i} x_{i}\right)}{P_{I}+Q_{I}} \\
=\frac{P_{I}\left[\frac{1}{P_{I}} \sum_{i \in I} p_{i} f\left(x_{i}\right)-f\left(\frac{1}{P_{I}} \sum_{i \in I} p_{i} x_{i}\right)\right]}{P_{I}+Q_{I}} \\
\quad+\frac{Q_{I}\left[\frac{1}{Q_{I}} \sum_{i \in I} q_{i} f\left(x_{i}\right)-f\left(\frac{1}{Q_{I}} \sum_{i \in I} q_{i} x_{i}\right)\right]}{P_{I}+Q_{I}} .
\end{aligned}
$$

Since $\Phi$ is monotonic nondecreasing and concave, then by (2.4),

$$
\begin{gathered}
\Phi\left[\frac{1}{P_{I}+Q_{I}} \sum_{i \in I}\left(p_{i}+q_{i}\right) f\left(x_{i}\right)-f\left(\frac{1}{P_{I}+Q_{I}} \sum_{i \in I}\left(p_{i}+q_{i}\right) x_{i}\right)\right] \\
\geq \frac{P_{I} \Phi\left[\frac{1}{P_{I}} \sum_{i \in I} p_{i} f\left(x_{i}\right)-f\left(\frac{1}{P_{I}} \sum_{i \in I} p_{i} x_{i}\right)\right]}{P_{I}+Q_{I}} \\
+\frac{Q_{I} \Phi\left[\frac{1}{Q_{I}} \sum_{i \in I} q_{i} f\left(x_{i}\right)-f\left(\frac{1}{Q_{I}} \sum_{i \in I} q_{i} x_{i}\right)\right]}{P_{I}+Q_{I}},
\end{gathered}
$$

which, by multiplication with $P_{I}+Q_{I}>0$, produces the desired result (2.2).

(ii) If $p \geq q$, then by (i),

$$
\begin{aligned}
D(f, I, p, x ; \Phi) & =D(f, I,(p-q)+q, x ; \Phi) \\
& \geq D(f, I, p-q, x ; \Phi)+D(f, I, p, x ; \Phi) \\
& \geq D(f, I, p, x ; \Phi)
\end{aligned}
$$

since $D(f, I, p-q, x ; \Phi) \geq 0$. 
Corollary 2.2. Let $f \in \operatorname{Conv}(C, \mathbb{R}), I \in P_{f}(\mathbb{N})$ and $x \in J_{*}(C)$. Assume that $\Phi$ : $[0, \infty) \rightarrow[0, \infty)$ is monotonic nondecreasing and concave.

If there exist the numbers $M \geq m \geq 0$ such that $M q \geq p \geq m q$, then

$$
\begin{aligned}
M Q_{I} & \Phi\left[\frac{1}{Q_{I}} \sum_{i \in I} q_{i} f\left(x_{i}\right)-f\left(\frac{1}{Q_{I}} \sum_{i \in I} q_{i} x_{i}\right)\right] \\
& \geq P_{I} \Phi\left[\frac{1}{P_{I}} \sum_{i \in I} p_{i} f\left(x_{i}\right)-f\left(\frac{1}{P_{I}} \sum_{i \in I} p_{i} x_{i}\right)\right] \\
& \geq m Q_{I} \Phi\left[\frac{1}{Q_{I}} \sum_{i \in I} q_{i} f\left(x_{i}\right)-f\left(\frac{1}{Q_{I}} \sum_{i \in I} q_{i} x_{i}\right)\right] .
\end{aligned}
$$

In particular,

$$
\begin{aligned}
\frac{M}{m} \Phi[ & {\left[\frac{1}{Q_{I}} \sum_{i \in I} q_{i} f\left(x_{i}\right)-f\left(\frac{1}{Q_{I}} \sum_{i \in I} q_{i} x_{i}\right)\right] } \\
& \geq \Phi\left[\frac{1}{P_{I}} \sum_{i \in I} p_{i} f\left(x_{i}\right)-f\left(\frac{1}{P_{I}} \sum_{i \in I} p_{i} x_{i}\right)\right] \\
& \geq \frac{m}{M} \Phi\left[\frac{1}{Q_{I}} \sum_{i \in I} q_{i} f\left(x_{i}\right)-f\left(\frac{1}{Q_{I}} \sum_{i \in I} q_{i} x_{i}\right)\right] .
\end{aligned}
$$

Now, if we write

$$
S(\mathbf{1}):=\left\{p \in J^{+}(\mathbb{R}): p_{i} \leq 1 \text { for all } i \in \mathbb{N}\right\}
$$

then we can state the following result as well.

Corollary 2.3. Let $f \in \operatorname{Conv}(C, \mathbb{R}), I \in P_{f}(\mathbb{N})$ and $x \in J_{*}(C)$. Assume that $\Phi$ : $[0, \infty) \rightarrow[0, \infty)$ is monotonic nondecreasing and concave. Then we have the bound

$$
\begin{aligned}
& \sup _{p \in S(\mathbf{1})}\left\{P_{I} \Phi\left[\frac{1}{P_{I}} \sum_{i \in I} p_{i} f\left(x_{i}\right)-f\left(\frac{1}{P_{I}} \sum_{i \in I} p_{i} x_{i}\right)\right]\right\} \\
& =\operatorname{card}(I) \Phi\left[\frac{1}{\operatorname{card}(I)} \sum_{i \in I} f\left(x_{i}\right)-f\left(\frac{1}{\operatorname{card}(I)} \sum_{i \in I} x_{i}\right)\right],
\end{aligned}
$$

where card $(I)$ denotes the cardinal of the finite set $I$.

REMARK 2.4. If we consider the concave and monotonic increasing function $\Phi(t)=$ $\ln t$ and assume that $f \in \operatorname{Conv}(C, \mathbb{R})$ and $x \in J_{*}(C)$ are selected such that

$$
\frac{1}{P_{I}} \sum_{i \in I} p_{i} f\left(x_{i}\right)>f\left(\frac{1}{P_{I}} \sum_{i \in I} p_{i} x_{i}\right)
$$


for any $I \in P_{f}(\mathbb{N})$ with $\operatorname{card}(I) \geq 2$ and $p \in J^{+}(\mathbb{R})$ (notice that it is enough to assume that $f$ is strictly convex and $x$ is not constant) then by the superadditivity of the functional

$$
\begin{aligned}
D(f, I, p, x ; \ln ) & :=P_{I} \ln \left[\frac{1}{P_{I}} \sum_{i \in I} p_{i} f\left(x_{i}\right)-f\left(\frac{1}{P_{I}} \sum_{i \in I} p_{i} x_{i}\right)\right] \\
& =\ln K(f, I, p, x),
\end{aligned}
$$

where

$$
K(f, I, p, x):=\left[\frac{1}{P_{I}} \sum_{i \in I} p_{i} f\left(x_{i}\right)-f\left(\frac{1}{P_{I}} \sum_{i \in I} p_{i} x_{i}\right)\right]^{P_{I}},
$$

we deduce that $K(f, I, \cdot, x)$ is supermultiplicative, that is, it satisfies the property

$$
K(f, I, p+q, x) \geq K(f, I, p, x) K(f, I, q, x)
$$

for any $p, q \in J^{+}(\mathbb{R})$.

The proof is obvious by the monotonicity and the positive homogeneity of the functional $D(f, I, \cdot, x ; \ln )$.

Note that inequality (2.9) has been obtained in a different way by Agarwal and Dragomir in [1].

Another important example of a concave and monotonic increasing function is $\Phi(t)=t^{s}$ with $s \in(0,1]$. In this situation the functional

$$
D_{s}(f, I, p, x):=\left[P_{I}^{s-1} \sum_{i \in I} p_{i} f\left(x_{i}\right)-P_{I}^{s} f\left(\frac{1}{P_{I}} \sum_{i \in I} p_{i} x_{i}\right)\right]^{s} \geq 0
$$

is superadditive and monotonic nondecreasing as a function of the weights $p$.

It might be useful for applications to observe that the superadditivity property is translated into the following version of Jensen's inequality:

$$
\begin{aligned}
{\left[\left(P_{I}+\right.\right.} & \left.\left.Q_{I}\right)^{s-1} \sum_{i \in I}\left(p_{i}+q_{i}\right) f\left(x_{i}\right)-\left(P_{I}+Q_{I}\right)^{s} f\left(\frac{\sum_{i \in I}\left(p_{i}+q_{i}\right) x_{i}}{P_{I}+Q_{I}}\right)\right]^{s} \\
\geq & {\left[P_{I}^{s-1} \sum_{i \in I} p_{i} f\left(x_{i}\right)-P_{I}^{s} f\left(\frac{1}{P_{I}} \sum_{i \in I} p_{i} x_{i}\right)\right]^{s} } \\
& +\left[Q_{I}^{s-1} \sum_{i \in I} q_{i} f\left(x_{i}\right)-Q_{I}^{s} f\left(\frac{1}{Q_{I}} \sum_{i \in I} q_{i} x_{i}\right)\right]^{s}(\geq 0)
\end{aligned}
$$

where $p, q \in J^{+}(\mathbb{R})$. 
The property of monotonicity provides the following double inequality for $p, q \in$ $J^{+}(\mathbb{R})$ such that $M q \geq p \geq m q$ and $M \geq m \geq 0$ :

$$
\begin{gathered}
M\left[Q_{I}^{s-1} \sum_{i \in I} q_{i} f\left(x_{i}\right)-Q_{I}^{s} f\left(\frac{1}{Q_{I}} \sum_{i \in I} q_{i} x_{i}\right)\right]^{s} \\
\geq\left[P_{I}^{s-1} \sum_{i \in I} p_{i} f\left(x_{i}\right)-P_{I}^{s} f\left(\frac{1}{P_{I}} \sum_{i \in I} p_{i} x_{i}\right)\right]^{s} \\
\geq m\left[Q_{I}^{s-1} \sum_{i \in I} q_{i} f\left(x_{i}\right)-Q_{I}^{s} f\left(\frac{1}{Q_{I}} \sum_{i \in I} q_{i} x_{i}\right)\right]^{s} .
\end{gathered}
$$

This inequality has the equivalent form

$$
\begin{aligned}
M^{1 / s} & {\left[Q_{I}^{s-1} \sum_{i \in I} q_{i} f\left(x_{i}\right)-Q_{I}^{s} f\left(\frac{1}{Q_{I}} \sum_{i \in I} q_{i} x_{i}\right)\right] } \\
& \geq P_{I}^{s-1} \sum_{i \in I} p_{i} f\left(x_{i}\right)-P_{I}^{s} f\left(\frac{1}{P_{I}} \sum_{i \in I} p_{i} x_{i}\right) \\
& \geq m^{1 / s}\left[Q_{I}^{s-1} \sum_{i \in I} q_{i} f\left(x_{i}\right)-Q_{I}^{s} f\left(\frac{1}{Q_{I}} \sum_{i \in I} q_{i} x_{i}\right)\right] .
\end{aligned}
$$

Finally, from the Corollary 2.3 we also have the bound

$$
\begin{aligned}
& \sup _{p \in S(\mathbf{1})}\left\{P_{I}^{s-1} \sum_{i \in I} p_{i} f\left(x_{i}\right)-P_{I}^{s} f\left(\frac{1}{P_{I}} \sum_{i \in I} p_{i} x_{i}\right)\right\} \\
& =[\operatorname{card}(I)]^{s-1} \sum_{i \in I} f\left(x_{i}\right)-[\operatorname{card}(I)]^{s} f\left(\frac{1}{\operatorname{card}(I)} \sum_{i \in I} x_{i}\right) .
\end{aligned}
$$

For a function $\Psi:(0, \infty) \rightarrow(0, \infty)$ we now consider the functional

$$
\begin{aligned}
D(f, I, p, x ; \Phi, \Psi):= & \sum_{i \in I} \Psi\left(p_{i}\right) \Phi\left[\frac{1}{\sum_{i \in I} \Psi\left(p_{i}\right)} \sum_{i \in I} \Psi\left(p_{i}\right) f\left(x_{i}\right)\right. \\
& \left.-f\left(\frac{1}{\sum_{i \in I} \Psi\left(p_{i}\right)} \sum_{i \in I} \Psi\left(p_{i}\right) x_{i}\right)\right]
\end{aligned}
$$

where $f \in \operatorname{Conv}(C, \mathbb{R}), I \in P_{f}(\mathbb{N}), p \in J^{+}(\mathbb{R}), x \in J_{*}(C)$. Now, if we denote by $\Psi(p)$ the sequence $\left\{\Psi\left(p_{i}\right)\right\}_{i \in \mathbb{N}}$, then we observe that

$$
D(f, I, p, x ; \Phi, \Psi)=D(f, I, \Psi(p), x ; \Phi) .
$$

The following result may be stated.

Corollary 2.5. Let $f \in \operatorname{Conv}(C, \mathbb{R}), I \in P_{f}(\mathbb{N})$ and $x \in J_{*}(C)$. Assume that $\Phi$ : $[0, \infty) \rightarrow[0, \infty)$ is monotonic nondecreasing and concave. If $\Psi:(0, \infty) \rightarrow(0, \infty)$ is concave, then $D(f, I, \cdot, x ; \Phi, \Psi)$ is also concave on $J^{+}(\mathbb{R})$. 
PROOF. Utilizing the properties of monotonicity, superadditivity and positive homogeneity of the functional $D(f, I, \cdot, x ; \Phi)$, we may successively write

$$
\begin{aligned}
D(f, & I, t p+(1-t) q, x ; \Phi, \Psi)=D(f, I, \Psi(t p+(1-t) q), x ; \Phi) \\
& \geq D(f, I, t \Psi(p)+(1-t) \Psi(q), x ; \Phi) \\
& \geq D(f, I, t \Psi(p), x ; \Phi)+D(f, I,(1-t) \Psi(q), x ; \Phi) \\
& =t D(f, I, \Psi(p), x ; \Phi)+(1-t) D(f, I, \Psi(q), x ; \Phi) \\
& =t D(f, I, p, x ; \Phi, \Psi)+(1-t) D(f, I, p, x ; \Phi, \Psi)
\end{aligned}
$$

for any $p, q \in J^{+}(\mathbb{R})$ and $t \in[0,1]$, which proves the statement.

\section{Some superadditivity properties for the index}

The following result concerning the superadditivity and monotonicity of the functional $D$ as an index set function holds.

Theorem 3.1. Let $f \in \operatorname{Conv}(C, \mathbb{R}), p \in J^{+}(\mathbb{R})$ and $x \in J_{*}(C)$. Assume that $\Phi$ : $[0, \infty) \rightarrow \mathbb{R}$ is monotonic nondecreasing and concave

(i) If $I, H \in P_{f}(\mathbb{N})$ with $I \cap H=\varnothing$, then

$$
D(f, I \cup H, p, x ; \Phi) \geq D(f, I, p, x ; \Phi)+D(f, H, p, x ; \Phi),
$$

that is, $D(f, \cdot, p, x ; \Phi)$ is superadditive as an index set function on $P_{f}(\mathbb{N})$.

(ii) If I, $H \in P_{f}(\mathbb{N})$ with $H \subset I$ and $\Phi:[0, \infty) \rightarrow[0, \infty)$, then

$$
D(f, I, p, x ; \Phi) \geq D(f, H, p, x ; \Phi)(\geq 0),
$$

that is, $D(f, \cdot, p, x ; \Phi)$ is monotonic nondecreasing as an index set function on $P_{f}(\mathbb{N})$.

\section{PROOF.}

(i) Let $I, H \in P_{f}(\mathbb{N})$ with $I \cap H=\varnothing$. By the convexity of the function $f$ on $C$ we have

$$
\begin{aligned}
\frac{1}{P_{I \cup H}} & \sum_{k \in I \cup H} p_{k} f\left(x_{k}\right)-f\left(\frac{1}{P_{I \cup H}} \sum_{k \in I \cup H} p_{k} x_{k}\right) \\
= & \frac{P_{I}\left(\frac{1}{P_{I}} \sum_{i \in I} p_{i} f\left(x_{i}\right)\right)+P_{H}\left(\frac{1}{P_{H}} \sum_{j \in H} p_{j} f\left(x_{j}\right)\right)}{P_{I}+P_{H}} \\
& -f\left(\frac{P_{I}\left(\frac{1}{P_{I}} \sum_{i \in I} p_{i} x_{i}\right)+P_{H}\left(\frac{1}{P_{H}} \sum_{j \in H} p_{j} x_{j}\right)}{P_{I}+P_{H}}\right) \\
\geq & \frac{P_{I}\left(\frac{1}{P_{I}} \sum_{i \in I} p_{i} f\left(x_{i}\right)\right)+P_{H}\left(\frac{1}{P_{H}} \sum_{j \in H} p_{j} f\left(x_{j}\right)\right)}{P_{I}+P_{H}}
\end{aligned}
$$




$$
\begin{aligned}
& -\frac{P_{I} f\left(\frac{1}{P_{I}} \sum_{i \in I} p_{i} x_{i}\right)+P_{H} f\left(\frac{1}{P_{H}} \sum_{j \in H} p_{j} x_{j}\right)}{P_{I}+P_{H}} \\
= & \frac{P_{I}\left[\frac{1}{P_{I}} \sum_{i \in I} p_{i} f\left(x_{i}\right)-f\left(\frac{1}{P_{I}} \sum_{i \in I} p_{i} x_{i}\right)\right]}{P_{I}+P_{H}} \\
& +\frac{P_{H}\left[\frac{1}{P_{H}} \sum_{j \in H} p_{j} f\left(x_{j}\right)-f\left(\frac{1}{P_{H}} \sum_{j \in H} p_{j} x_{j}\right)\right]}{P_{I}+P_{H}} .
\end{aligned}
$$

Since $\Phi$ is monotonic nondecreasing and concave, then by (3.3),

$$
\begin{aligned}
\Phi\left[\frac{1}{P_{I \cup H}} \sum_{k \in I \cup H} p_{k} f\left(x_{k}\right)-f\left(\frac{1}{P_{I \cup H}} \sum_{k \in I \cup H} p_{k} x_{k}\right)\right] \\
\geq \\
\quad \frac{P_{I} \Phi\left[\frac{1}{P_{I}} \sum_{i \in I} p_{i} f\left(x_{i}\right)-f\left(\frac{1}{P_{I}} \sum_{i \in I} p_{i} x_{i}\right)\right]}{P_{I}+P_{H}} \\
\quad+\frac{P_{H} \Phi\left[\frac{1}{P_{H}} \sum_{j \in H} p_{j} f\left(x_{j}\right)-f\left(\frac{1}{P_{H}} \sum_{j \in H} p_{j} x_{j}\right)\right]}{P_{I}+P_{H}},
\end{aligned}
$$

which, by multiplication with $P_{I}+P_{H}>0$, produces the desired result (3.2).

(ii) If $I, H \in P_{f}(\mathbb{N})$ with $H \subset I$, then

$$
\begin{aligned}
D(f, I, p, x ; \Phi) & =D(f,(I \backslash H) \cup H, p, x ; \Phi) \\
& \geq D(f, I \backslash H, p, x ; \Phi)+D(f, H, p, x ; \Phi) \\
& \geq D(f, H, p, x ; \Phi)(\geq 0)
\end{aligned}
$$

since $D(f, I \backslash H, p, x ; \Phi) \geq 0$.

For the special case $I=I_{n}:=\{1, \ldots, n\}$ we write $D_{n}(f, p, x ; \Phi)$ instead of $D\left(f, I_{n}, p, x ; \Phi\right)$, that is,

$$
D_{n}(f, p, x ; \Phi)=P_{n} \Phi\left[\frac{1}{P_{n}} \sum_{i=1}^{n} p_{i} f\left(x_{i}\right)-f\left(\frac{1}{P_{n}} \sum_{i=1}^{n} p_{i} x_{i}\right)\right]
$$

where $P_{n}=P_{I_{n}}=\sum_{i=1}^{n} p_{i}>0$.

The following particular case is of interest.

Corollary 3.2. Let $f \in \operatorname{Conv}(C, \mathbb{R}), p \in J^{+}(\mathbb{R})$ and $x \in J_{*}(C)$. Assume that $\Phi$ : $[0, \infty) \rightarrow[0, \infty)$ is monotonic nondecreasing and concave. Then

$$
\max _{I \sqsubseteq I_{n}} D(f, I, p, x ; \Phi)=D_{n}(f, p, x ; \Phi) \geq 0,
$$

$$
\begin{aligned}
& D_{n}(f, p, x ; \Phi) \\
& \left.\quad \geq \max _{1 \leq i<j \leq n}\left\{\left(p_{i}+p_{j}\right) \Phi\left[\frac{p_{i} f\left(x_{i}\right)+p_{j} f\left(x_{j}\right)}{p_{i}+p_{j}}-f\left(\frac{p_{i} x_{i}+p_{j} x_{j}}{p_{i}+p_{j}}\right)\right]\right\} \geq 0^{(3 .} .6\right)
\end{aligned}
$$


and

$$
D_{n}(f, p, x ; \Phi) \geq D_{n-1}(f, p, x ; \Phi) \geq \cdots \geq D_{2}(f, p, x ; \Phi) \geq 0 .
$$

This is obvious by the monotonicity property of the functional $D(f, \cdot, p, x ; \Phi)$ as an index set function.

If we use the superadditivity property, then we can state the following result as well. Corollary 3.3. Let $f \in \operatorname{Conv}(C, \mathbb{R}), p \in J^{+}(\mathbb{R})$ and $x \in J_{*}(C)$. Assume that $\Phi$ : $[0, \infty) \rightarrow \mathbb{R}$ is monotonic nondecreasing and concave. Then

$$
\begin{aligned}
P_{2 n} \Phi[ & \left.\frac{1}{P_{2 n}} \sum_{i=1}^{2 n} p_{i} f\left(x_{i}\right)-f\left(\frac{1}{P_{2 n}} \sum_{i=1}^{2 n} p_{i} x_{i}\right)\right] \\
\geq & \sum_{i=1}^{n} p_{2 i} \Phi\left[\frac{1}{\sum_{i=1}^{n} p_{2 i}} \sum_{i=1}^{n} p_{2 i} f\left(x_{2 i}\right)-f\left(\frac{1}{\sum_{i=1}^{n} p_{2 i}} \sum_{i=1}^{n} p_{2 i} x_{2 i}\right)\right] \\
& +\sum_{i=1}^{n} p_{2 i-1} \Phi\left[\frac{1}{\sum_{i=1}^{n} p_{2 i-1}} \sum_{i=1}^{n} p_{2 i-1} f\left(x_{2 i-1}\right)\right. \\
& \left.-f\left(\frac{1}{\sum_{i=1}^{n} p_{2 i-1}} \sum_{i=1}^{n} p_{2 i-1} x_{2 i-1}\right)\right]
\end{aligned}
$$

and

$$
\begin{aligned}
P_{2 n+1} \Phi & {\left[\frac{1}{P_{2 n+1}} \sum_{i=1}^{2 n+1} p_{i} f\left(x_{i}\right)-f\left(\frac{1}{P_{2 n+1}} \sum_{i=1}^{2 n+1} p_{i} x_{i}\right)\right] } \\
\geq & \sum_{i=1}^{n} p_{2 i} \Phi\left[\frac{1}{\sum_{i=1}^{n} p_{2 i}} \sum_{i=1}^{n} p_{2 i} f\left(x_{2 i}\right)-f\left(\frac{1}{\sum_{i=1}^{n} p_{2 i}} \sum_{i=1}^{n} p_{2 i} x_{2 i}\right)\right] \\
& +\sum_{i=1}^{n} p_{2 i+1} \Phi\left[\frac{1}{\sum_{i=1}^{n} p_{2 i+1}} \sum_{i=1}^{n} p_{2 i+1} f\left(x_{2 i+1}\right)\right. \\
& \left.-f\left(\frac{1}{\sum_{i=1}^{n} p_{2 i+1}} \sum_{i=1}^{n} p_{2 i+1} x_{2 i+1}\right)\right] .
\end{aligned}
$$

REMARK 3.4. If we consider the functional defined in (2.7), namely

$$
K(f, I, p, x):=\left[\frac{1}{P_{I}} \sum_{i \in I} p_{i} f\left(x_{i}\right)-f\left(\frac{1}{P_{I}} \sum_{i \in I} p_{i} x_{i}\right)\right]^{P_{I}},
$$

then by Theorem 3.1,

$$
K(f, I \cup H, p, x) \geq K(f, I, p, x) \cdot K(f, H, p, x)
$$

for any $I, H \in P_{f}(\mathbb{N})$ with $I \cap H=\varnothing$, meaning that the functional $K(f, \cdot, p, x)$ is supermultiplicative as an index set mapping. 
This fact obviously implies the following multiplicative inequalities of interest:

$$
\begin{aligned}
{\left[\frac{1}{P_{2 n}} \sum_{i=1}^{2 n} p_{i} f\left(x_{i}\right)-f\left(\frac{1}{P_{2 n}} \sum_{i=1}^{2 n} p_{i} x_{i}\right)\right]^{P_{2 n}} } \\
\geq\left[\frac{1}{\sum_{i=1}^{n} p_{2 i}} \sum_{i=1}^{n} p_{2 i} f\left(x_{2 i}\right)-f\left(\frac{1}{\sum_{i=1}^{n} p_{2 i}} \sum_{i=1}^{n} p_{2 i} x_{2 i}\right)\right]^{\sum_{i=1}^{n} p_{2 i}} \\
\quad \times\left[\frac{1}{\sum_{i=1}^{n} p_{2 i-1}} \sum_{i=1}^{n} p_{2 i-1} f\left(x_{2 i-1}\right)\right. \\
\left.\quad-f\left(\frac{1}{\sum_{i=1}^{n} p_{2 i-1}} \sum_{i=1}^{n} p_{2 i-1} x_{2 i-1}\right)\right]^{\sum_{i=1}^{n} p_{2 i-1}}
\end{aligned}
$$

where $f \in \operatorname{Conv}(C, \mathbb{R}), p \in J^{+}(\mathbb{R})$ and $x \in J_{*}(C)$.

Moreover, if we consider the functional defined in (2.10) by

$$
D_{s}(f, I, p, x):=\left[P_{I}^{s-1} \sum_{i \in I} p_{i} f\left(x_{i}\right)-P_{I}^{s} f\left(\frac{1}{P_{I}} \sum_{i \in I} p_{i} x_{i}\right)\right]^{s} \geq 0
$$

where $s \in(0,1]$ and introduce the associated functional

$$
F_{S}(f, I, p, x):=P_{I}^{s-1} \sum_{i \in I} p_{i} f\left(x_{i}\right)-P_{I}^{s} f\left(\frac{1}{P_{I}} \sum_{i \in I} p_{i} x_{i}\right)
$$

then by denoting

$$
F_{s, n}(f, p, x):=F_{s}\left(f, I_{n}, p, x\right)=P_{n}^{s-1} \sum_{i=1}^{n} p_{i} f\left(x_{i}\right)-P_{n}^{s} f\left(\frac{1}{P_{n}} \sum_{i=1}^{n} p_{i} x_{i}\right)
$$

where $I_{n}=\{1, \ldots, n\}$, we have that the sequence $\left\{F_{s, n}(f, p, x)\right\}_{n \geq 2}$ is nondecreasing and the following bounds are valid:

$$
\max _{I \sqsubseteq I_{n}} F_{S}(f, I, p, x)=F_{s, n}(f, p, x)
$$

and

$$
\begin{aligned}
F_{s, n}(f, p, x) & \geq \max _{1 \leq i<j \leq n}\left\{\frac{p_{i} f\left(x_{i}\right)+p_{j} f\left(x_{j}\right)}{\left(p_{i}+p_{j}\right)^{1-s}}-\left(p_{i}+p_{j}\right)^{s} f\left(\frac{p_{i} x_{i}+p_{j} x_{j}}{p_{i}+p_{j}}\right)\right\} \\
& \geq 0 .
\end{aligned}
$$




\section{Applications for norm inequalities}

Let $(X,\|\cdot\|)$ be a real or complex normed linear space. It is well known that the function $f_{p}: X \rightarrow \mathbb{R}, f_{p}(x)=\|x\|^{p}, p \geq 1$, is convex on $X$. Assume that $p=\left(p_{1}, \ldots, p_{n}\right)$ and $q=\left(q_{1}, \ldots, q_{n}\right)$ are probability distributions with all $q_{j}$ nonzero. In [10] we obtained the following refinements of the generalized triangle inequality:

$$
\begin{gathered}
\max _{1 \leq i \leq n}\left\{\frac{p_{i}}{q_{i}}\right\}\left[\sum_{j=1}^{n} q_{j}\left\|x_{j}\right\|^{p}-\left\|\sum_{j=1}^{n} q_{j} x_{j}\right\|^{p}\right] \geq \sum_{j=1}^{n} p_{j}\left\|x_{j}\right\|^{p}-\left\|\sum_{j=1}^{n} p_{j} x_{j}\right\|^{p} \\
\geq \min _{1 \leq i \leq n}\left\{\frac{p_{i}}{q_{i}}\right\}\left[\sum_{j=1}^{n} q_{j}\left\|x_{j}\right\|^{p}-\left\|\sum_{j=1}^{n} q_{j} x_{j}\right\|^{p}\right](\geq 0)
\end{gathered}
$$

and

$$
\begin{gathered}
\max _{1 \leq i \leq n}\left\{p_{i}\right\}\left[\sum_{j=1}^{n}\left\|x_{j}\right\|^{p}-n^{1-p}\left\|\sum_{j=1}^{n} x_{j}\right\|^{p}\right] \geq \sum_{j=1}^{n} p_{j}\left\|x_{j}\right\|^{p}-\left\|\sum_{j=1}^{n} p_{j} x_{j}\right\|^{p} \\
\geq \min _{1 \leq i \leq n}\left\{p_{i}\right\}\left[\sum_{j=1}^{n}\left\|x_{j}\right\|^{p}-n^{1-p}\left\|\sum_{j=1}^{n} x_{j}\right\|^{p}\right](\geq 0)
\end{gathered}
$$

for all $p \geq 1$.

We remark that, for $p=1$ one may obtain from the previous results the following inequalities that are intimately related with the generalized triangle inequality in normed spaces:

$$
\begin{gathered}
\max _{1 \leq i \leq n}\left\{\frac{p_{i}}{q_{i}}\right\}\left[\sum_{j=1}^{n} q_{j}\left\|x_{j}\right\|-\left\|\sum_{j=1}^{n} q_{j} x_{j}\right\|\right] \geq \sum_{j=1}^{n} p_{j}\left\|x_{j}\right\|-\left\|\sum_{j=1}^{n} p_{j} x_{j}\right\| \\
\geq \min _{1 \leq i \leq n}\left\{\frac{p_{i}}{q_{i}}\right\}\left[\sum_{j=1}^{n} q_{j}\left\|x_{j}\right\|-\left\|\sum_{j=1}^{n} q_{j} x_{j}\right\|\right](\geq 0), \\
\max _{1 \leq i \leq n}\left\{p_{i}\right\}\left[\sum_{j=1}^{n}\left\|x_{j}\right\|-\left\|\sum_{j=1}^{n} x_{j}\right\|\right] \geq \sum_{j=1}^{n} p_{j}\left\|x_{j}\right\|-\left\|\sum_{j=1}^{n} p_{j} x_{j}\right\| \\
\geq \min _{1 \leq i \leq n}\left\{p_{i}\right\}\left[\sum_{j=1}^{n}\left\|x_{j}\right\|-\left\|\sum_{j=1}^{n} x_{j}\right\|\right](\geq 0) .
\end{gathered}
$$

If in (4.4) we take

$$
p_{j}:=\frac{1 /\left\|x_{j}\right\|}{\sum_{k=1}^{n}\left(1 /\left\|x_{k}\right\|\right)}, \quad \text { with } x_{j} \neq 0 \forall j \in\{1, \ldots, n\},
$$


then, by rearranging the inequality, we get the result:

$$
\begin{gathered}
\max _{1 \leq j \leq n}\left\{\left\|x_{j}\right\|\right\}\left[n-\left\|\sum_{j=1}^{n} \frac{x_{j}}{\left\|x_{j}\right\|}\right\|\right] \geq \sum_{j=1}^{n}\left\|x_{j}\right\|-\left\|\sum_{j=1}^{n} x_{j}\right\| \\
\geq \min _{1 \leq j \leq n}\left\{\left\|x_{j}\right\|\right\}\left[n-\left\|\sum_{j=1}^{n} \frac{x_{j}}{\left\|x_{j}\right\|}\right\|\right] .
\end{gathered}
$$

We note that inequality (4.5) has been obtained in a different way by Kato et al. in [16] where an analysis of the equality case for strictly convex spaces has been performed as well.

We can state the following result which provides a generalization of inequality (4.1).

Proposition 4.1. Let $(X,\|\cdot\|)$ be a normed linear space, $x=\left(x_{1}, \ldots, x_{n}\right)$ an $n$-tuple of vectors in $X$, and $p=\left(p_{1}, \ldots, p_{n}\right)$ and $q=\left(q_{1}, \ldots, q_{n}\right)$ probability distributions with all $q_{j}$ nonzero. If $t \geq 1$ and $\Phi:[0, \infty) \rightarrow[0, \infty)$ is monotonic nondecreasing and concave, then

$$
\begin{aligned}
\max _{1 \leq i \leq n} & \left\{\frac{p_{i}}{q_{i}}\right\} \Phi\left[\sum_{i=1}^{n} q_{i}\left\|x_{i}\right\|^{t}-\left\|\sum_{i=1}^{n} q_{i} x_{i}\right\|^{t}\right] \\
& \geq \Phi\left[\sum_{i=1}^{n} p_{i}\left\|x_{i}\right\|^{t}-\left\|\sum_{i=1}^{n} p_{i} x_{i}\right\|^{t}\right] \\
& \geq \min _{1 \leq i \leq n}\left\{\frac{p_{i}}{q_{i}}\right\} \Phi\left[\sum_{i=1}^{n} q_{i}\left\|x_{i}\right\|^{t}-\left\|\sum_{i=1}^{n} q_{i} x_{i}\right\|^{t}\right]
\end{aligned}
$$

and, in particular,

$$
\begin{aligned}
& n \max _{1 \leq i \leq n}\left\{p_{i}\right\} \Phi\left[n^{-1} \sum_{i=1}^{n}\left\|x_{i}\right\|^{t}-n^{-t}\left\|\sum_{i=1}^{n} x_{i}\right\|^{t}\right] \\
& \geq \Phi\left[\sum_{i=1}^{n} p_{i}\left\|x_{i}\right\|^{t}-\left\|\sum_{i=1}^{n} q_{i} x_{i}\right\|^{t}\right] \\
& \quad \geq n \min _{1 \leq i \leq n}\left\{p_{i}\right\} \Phi\left[n^{-1} \sum_{i=1}^{n}\left\|x_{i}\right\|^{t}-n^{-t}\left\|\sum_{i=1}^{n} x_{i}\right\|^{t}\right] .
\end{aligned}
$$

The proof follows from Corollary 2.2 and the details are omitted.

Now, if $p=\left(p_{1}, \ldots, p_{n}\right)$ are positive weights with $P_{n}=\sum_{i=1}^{n} p_{i}>0$ and $x=$ $\left(x_{1}, \ldots, x_{n}\right)$ is an $n$-tuple of vectors in $X$, then by defining the functional

$$
D_{n}(t,\|\cdot\|, p, x ; \Phi)=P_{n} \Phi\left[P_{n}^{-1} \sum_{i=1}^{n} p_{i}\left\|x_{i}\right\|^{t}-P_{n}^{-t}\left\|\sum_{i=1}^{n} p_{i} x_{i}\right\|^{t}\right]
$$

we can state the following result as well. 
Proposition 4.2. If $t \geq 1$ and $\Phi:[0, \infty) \rightarrow[0, \infty)$ is monotonic nondecreasing and concave, then

$$
\begin{aligned}
& D_{n}(t,\|\cdot\|, p, x ; \Phi) \\
& \quad \geq \max _{1 \leq i<j \leq n}\left\{\left(p_{i}+p_{j}\right) \Phi\left[\frac{p_{i}\left\|x_{i}\right\|^{t}+p_{j}\left\|x_{j}\right\|^{t}}{p_{i}+p_{j}}-\left\|\frac{p_{i} x_{i}+p_{j} x_{j}}{p_{i}+p_{j}}\right\|^{t}\right]\right\} \geq 0
\end{aligned}
$$

and

$$
D_{n}(t,\|\cdot\|, p, x ; \Phi) \geq D_{n-1}(t,\|\cdot\|, p, x ; \Phi) \geq \cdots \geq D_{2}(t,\|\cdot\|, p, x ; \Phi) \geq 0 .
$$

The proof follows from Corollary 3.2 and the details are omitted.

\section{Applications for $f$-divergences}

Given a convex function $f:[0, \infty) \rightarrow \mathbb{R}$, the $f$-divergence functional

$$
I_{f}(p, q)=\sum_{i=1}^{n} q_{i} f\left(\frac{p_{i}}{q_{i}}\right)
$$

was introduced by Csiszár [3] as a generalized measure of information, a 'distance function' on the set of probability distributions $\mathbb{P}^{n}$. The restriction here to discrete distributions is only for convenience; similar results hold for general distributions. As in Csiszár [3], we interpret undefined expressions as follows:

$$
\begin{gathered}
f(0)=\lim _{t \rightarrow 0+} f(t), \quad 0 f\left(\frac{0}{0}\right)=0, \\
0 f\left(\frac{a}{0}\right)=\lim _{\varepsilon \rightarrow 0+} \varepsilon f\left(\frac{a}{\varepsilon}\right)=a \lim _{t \rightarrow \infty} \frac{f(t)}{t}, \quad a>0 .
\end{gathered}
$$

The following results were essentially given by Csiszár and Körner [4].

Proposition 5.1 (Joint convexity). If $f:[0, \infty) \rightarrow \mathbb{R}$ is convex, then $I_{f}(p, q)$ is jointly convex in $p$ and $q$.

Proposition 5.2 (Jensen's inequality). Let $f:[0, \infty) \rightarrow \mathbb{R}$ be convex. Then for any $p, q \in[0, \infty)^{n}$ with $P_{n}:=\sum_{i=1}^{n} p_{i}>0, Q_{n}:=\sum_{i=1}^{n} q_{i}>0$, we have the inequality

$$
I_{f}(p, q) \geq Q_{n} f\left(\frac{P_{n}}{Q_{n}}\right) .
$$

If $f$ is strictly convex, equality holds in (5.2) if and only if

$$
\frac{p_{1}}{q_{1}}=\frac{p_{2}}{q_{2}}=\cdots=\frac{p_{n}}{q_{n}}
$$


It is natural to consider the following corollary.

Corollary 5.3 (Nonnegativity). Let $f:[0, \infty) \rightarrow \mathbb{R}$ be convex and normalized, that is,

$$
f(1)=0 .
$$

Then for any $p, q \in[0, \infty)^{n}$ with $P_{n}=Q_{n}$, we have the inequality

$$
I_{f}(p, q) \geq 0 .
$$

If $f$ is strictly convex, equality holds in (5.4) if and only if

$$
p_{i}=q_{i} \text { for all } i \in\{1, \ldots, n\} .
$$

In particular, if $p, q$ are probability vectors, then Corollary 5.3 shows, for strictly convex and normalized $f:[0, \infty) \rightarrow \mathbb{R}$, that

$$
I_{f}(p, q) \geq 0 \text { and } I_{f}(p, q)=0 \text { if and only if } p=q .
$$

We now give some examples of divergence measures in information theory which are particular cases of $f$-divergences.

Kullback-Leibler distance [18]. The Kullback-Leibler distance $D(\cdot, \cdot)$ is defined by

$$
D(p, q):=\sum_{i=1}^{n} p_{i} \log \left(\frac{p_{i}}{q_{i}}\right) .
$$

If we choose $f(t)=t \ln t, t>0$, then obviously

$$
I_{f}(p, q)=D(p, q) .
$$

Variational distance $\left(\boldsymbol{l}_{\mathbf{1}}\right.$-distance). The variational distance $V(\cdot, \cdot)$ is defined by

$$
V(p, q):=\sum_{i=1}^{n}\left|p_{i}-q_{i}\right| .
$$

If we choose $f(t)=|t-1|, t \in[0, \infty)$, then

$$
I_{f}(p, q)=V(p, q)
$$

Hellinger discrimination [2]. The Hellinger discrimination is defined by $\sqrt{2 h^{2}(\cdot, \cdot)}$, where $h^{2}(\cdot, \cdot)$ is given by

$$
h^{2}(p, q):=\frac{1}{2} \sum_{i=1}^{n}\left(\sqrt{p_{i}}-\sqrt{q_{i}}\right)^{2} .
$$

It is obvious that if $f(t)=\frac{1}{2}(\sqrt{t}-1)^{2}$, then

$$
I_{f}(p, q)=h^{2}(p, q) .
$$


Triangular discrimination [22]. We define triangular discrimination between $p$ and $q$ by

$$
\Delta(p, q)=\sum_{i=1}^{n} \frac{\left|p_{i}-q_{i}\right|^{2}}{p_{i}+q_{i}} .
$$

It is obvious that if $f(t)=(t-1)^{2} /(t+1), t \in(0, \infty)$, then

$$
I_{f}(p, q)=\Delta(p, q) \text {. }
$$

Note that $\sqrt{\Delta(p, q)}$ is known in the literature as the Le Cam distance.

Chi-square distance. We define the chi-square $\left(\chi^{2}\right)$ distance by

$$
D_{\chi^{2}}(p, q):=\sum_{i=1}^{n} \frac{\left(p_{i}-q_{i}\right)^{2}}{q_{i}} .
$$

It is clear that if $f(t)=(t-1)^{2}, t \in[0, \infty)$, then

$$
I_{f}(p, q)=D_{\chi^{2}}(p, q) .
$$

Rényi's divergences [21]. For $\alpha \in \mathbb{R} \backslash\{0,1\}$, consider

$$
\rho_{\alpha}(p, q):=\sum_{i=1}^{n} p_{i}^{\alpha} q_{i}^{1-\alpha} .
$$

It is obvious that if $f(t)=t^{\alpha}(t \in(0, \infty))$, then

$$
I_{f}(p, q)=\rho_{\alpha}(p, q) .
$$

Rényi's divergences $R_{\alpha}(p, q):=1 /(\alpha(\alpha-1)) \ln \left[\rho_{\alpha}(p, q)\right]$ have been introduced for all real orders $\alpha \neq 0, \alpha \neq 1$ (and continuously extended for $\alpha=0$ and $\alpha=1$ ) in [19], where the reader may find many inequalities valid for these divergences, without, as well as with, restrictions for $p$ and $q$.

For other examples of divergence measures, see the paper [15] and the books [19, 23], where further references are given.

For a function $f:(0, \infty) \rightarrow \mathbb{R}$ we denote by $f^{\#}$ the function defined on $(0, \infty)$ by the equation $f^{\#}(x):=f(1 / x)$. With this notation,

$$
I_{f^{\#}}(p, q)=\sum_{i=1}^{n} q_{i} f^{\#}\left(\frac{p_{i}}{q_{i}}\right)=\sum_{i=1}^{n} q_{i} f\left(\frac{q_{i}}{p_{i}}\right) .
$$

Using Corollary 2.2, we can state the following result for $f$-divergences.

Proposition 5.4. Let $f:[0, \infty) \rightarrow \mathbb{R}$ be convex and normalized and $p, q$ two probability distributions such that $R:=\max _{i \in\{1, \ldots, n\}}\left\{p_{i} / q_{i}\right\}<\infty$ and $r:=\min _{i \in\{1, \ldots, n\}}$ $\left\{p_{i} / q_{i}\right\}>0$. If $\Phi:[0, \infty) \rightarrow[0, \infty)$ is monotonic nondecreasing and concave, then

$$
\begin{gathered}
R \Phi\left[I_{f^{\#}}(p, q)-f\left(D_{\chi^{2}}(q, p)+1\right)\right] \geq \Phi\left[I_{f}(q, p)\right] \\
\geq r \Phi\left[I_{f^{\#}}(p, q)-f\left(D_{\chi^{2}}(q, p)+1\right)\right] .
\end{gathered}
$$


PROOF. Utilizing the inequality (2.5),

$$
\begin{gathered}
R \Phi\left[\sum_{i=1}^{n} q_{i} f\left(\frac{q_{i}}{p_{i}}\right)-f\left(\sum_{i=1}^{n} \frac{q_{i}^{2}}{p_{i}}\right)\right] \geq \Phi\left[\sum_{i=1}^{n} p_{i} f\left(\frac{q_{i}}{p_{i}}\right)-f(1)\right] \\
\geq r \Phi\left[\sum_{i=1}^{n} q_{i} f\left(\frac{q_{i}}{p_{i}}\right)-f\left(\sum_{i=1}^{n} \frac{q_{i}^{2}}{p_{i}}\right)\right] .
\end{gathered}
$$

Since

$$
\sum_{i=1}^{n} \frac{q_{i}^{2}}{p_{i}}=D_{\chi^{2}}(q, p)+1
$$

then by (5.8) we deduce the desired result (5.7).

Finally, by means of Corollary 3.2 we also obtain the following lower bound for the $f$-divergence.

Proposition 5.5. Let $f:[0, \infty) \rightarrow \mathbb{R}$ be convex and normalized and $p, q$ two probability distributions. If $\Phi:[0, \infty) \rightarrow[0, \infty)$ is monotonic nondecreasing and concave, then:

$\Phi\left[I_{f}(q, p)\right] \geq \max _{1 \leq i<j \leq n}\left\{\left(p_{i}+p_{j}\right) \Phi\left[\frac{p_{i} f\left(\frac{q_{i}}{p_{i}}\right)+p_{j} f\left(\frac{q_{j}}{p_{j}}\right)}{p_{i}+p_{j}}-f\left(\frac{q_{i}+q_{j}}{p_{i}+p_{j}}\right)\right]\right\} \geq 0$.

REMARK 5.6. If one chooses different examples of convex functions generating the particular divergences mentioned at the beginning of the section, that one can obtain various inequalities of interest. However, the details are not presented here.

\section{References}

[1] R. P. Agarwal and S. S. Dragomir, 'The property of supermultiplicity for some classical inequalities and applications', Comput. Math. Appl. 35(6) (1998), 105-118.

[2] R. Beran, 'Minimum Hellinger distance estimates for parametric models', Ann. Statist. 5 (1977), $445-463$.

[3] I. Csiszár, 'Information-type measures of differences of probability distributions and indirect observations', Studia Sci. Math. Hungar. 2 (1967), 299-318.

[4] I. Csiszár and J. Körner, Information Theory: Coding Theorems for Discrete Memoryless Systems (Academic Press, New York, 1981).

[5] S. S. Dragomir, 'An improvement of Jensen's inequality', Bull. Math. Soc. Sci. Math. Roumanie 34(4) (1990), 291-296.

[6] S. S. Dragomir, 'Some refinements of Ky Fan's inequality', J. Math. Anal. Appl. 163(2) (1992), 317-321.

[7] S. S. Dragomir, 'Some refinements of Jensen's inequality', J. Math. Anal. Appl. 168(2) (1992), 518-522.

[8] S. S. Dragomir, 'A further improvement of Jensen's inequality', Tamkang J. Math. 25(1) (1994), 29-36.

[9] S. S. Dragomir, 'A new improvement of Jensen's inequality', Indian J. Pure Appl. Math. 26(10) (1995), 959-968. 
[10] S. S. Dragomir, 'Bounds for the normalized Jensen functional', Bull. Aust. Math. Soc. 74(3) (2006), 471-478.

[11] S. S. Dragomir, 'A refinement of Jensen's inequality with applications for $f$-divergence measures', Res. Rep. Coll. 10 (2007), Preprint; Supp., Article 15.

[12] S. S. Dragomir and C. J. Goh, 'A counterpart of Jensen's discrete inequality for differentiable convex mappings and applications in information theory', Math. Comput. Modelling 24(2) (1996), $1-11$.

[13] S. S. Dragomir and N. M. Ionescu, 'Some converse of Jensen's inequality and applications', Rev. Anal. Numér. Théor. Approx. 23(1) (1994), 71-78.

[14] S. S. Dragomir, J. Pečarić and L. E. Persson, 'Properties of some functionals related to Jensen's inequality', Acta Math. Hungar. 70(1-2) (1996), 129-143.

[15] J. N. Kapur, 'A comparative assessment of various measures of directed divergence', Adv. Manag. Stud. 3(1) (1984), 1-16.

[16] M. Kato, K.-S. Saito and T. Tamura, 'Sharp triangle inequality and its reverse in Banach spaces', Math. Inequal. Appl. 10(2) (2007), 451-460.

[17] S. Kullback, Information Theory and Statistics (Wiley, New York, 1959).

[18] S. Kullback and R. A. Leibler, 'On information and sufficiency', Ann. Math. Statist. 22 (1951), 79-86.

[19] F. Liese and I. Vajda, Convex Statistical Distances (Teubner, Leipzig, 1987).

[20] J. Pečarić and S. S. Dragomir, 'A refinement of Jensen inequality and applications', Studia Univ. Babeş-Bolyai Math. 24(1) (1989), 15-19.

[21] A. Rényi, 'On measures of entropy and information', in: Proceedings of the Fourth Berkeley Symposium on Mathematical Statistics and Probability, Vol. 1, (ed. J. Neyman) (University of California Press, Berkeley, CA, 1961).

[22] F. Topsoe, 'Some inequalities for information divergence and related measures of discrimination', Res. Rep. Coll. RGMIA 2(1) (1999), 85-98.7

[23] I. Vajda, Theory of Statistical Inference and Information (Kluwer, Boston, MA, 1989).

[24] P. M. Vasić and Ž. Mijajlović, 'On an index set function connected with Jensen inequality', Univ. Beograd. Publ. Elektrotehn. Fak. Ser. Mat. Fiz. 544-576 (1976), 110-112.

S. S. DRAGOMIR, Research Group in Mathematical Inequalities and Applications, School of Engineering and Science, Victoria University, PO Box 14428, Melbourne, Victoria 8001, Australia e-mail: sever.dragomir@vu.edu.au 